\title{
OPTICAL TRANSITION RADIATION IN PRESENCE OF ACOUSTIC WAVES
}

\author{
A. R. Mkrtchyan, V. V. Parazian, A. A. Saharian \\ Institute of Applied Problems in Physics, \\ 25 Nersessian Street, 0014 Yerevan, Armenia
}

June 5,2018

\begin{abstract}
Transition radiation from relativistic electrons is investigated in an ultrasonic superlattice excited in a finite thickness plate. In the quasi-classical approximation formulae are derived for the vector potential of the electromagnetic field and for the spectral-angular distribution of the radiation intensity. The acoustic waves generate new resonance peaks in the spectral and angular distribution of the radiation intensity. The heights of the peaks can be tuned by choosing the parameters of the acoustic wave.
\end{abstract}

Keywords: Transition radiation; physical effects of ultrasonics.

PACS Nos.: 41.60.Dk, 43.35.-c

\section{Introduction}

Transition radiation is produced when a relativistic particle traverses an inhomogeneous medium. Such radiation has a number of remarkable properties and at present it has found many important applications. In particular, optical transition radiation from metallic targets is widely used for the measurement of transverse size, divergence and energy of electron and proton beams (see, e.g. [1] and references therein). The intensity of the transition radiation can be increased considerably by using the interference effects in periodic structures (for a review see Refs. [2]-[7]). From the point of view of controlling the parameters of the high-energy electromagnetic processes in a medium, it is of interest to investigate the influence of external fields, such as acoustic waves, temperature gradient etc., on the corresponding characteristics. The considerations of concrete processes, such as diffraction radiation [8], parametric X-radiation [9], channeling radiation [10], electron-positron pair creation by high-energy photons [11], bremsstrahlung of high-energy electrons [12], have shown that the external fields can essentially change the angular-frequency characteristics of the radiation intensities. Recently there has been broad interest in compact crystalline undulators with periodically deformed crystallographic planes as an efficient source of high energy photons [13] (for a review with a more complete list of references see [14]). In Ref. [15] we have considered the X-ray transition radiation from ultrarelativistic electrons in an ultrasonic superlattice excited in fused quartz plate. The radiation from a charged particle for a semi-infinite laminated medium has been recently discussed in [16]. The present paper deals with the optical transition radiation from relativistic electrons in a plate in presence of ultrasonic vibrations. 
The paper is organized as follows. In the next section we evaluate the vector potential for the electromagnetic field in the quasi-classical approximation. The radiation intensity is investigated in section 3. Special cases of the general formula are considered. In section 4 we present the results of the numerical evaluation of the radiation intensity for the case of a plate made of fused quartz. The main results of the paper are summarized in section 5 .

\section{Electromagnetic field}

Consider the transition radiation emerging in the forward direction when a charge $e$ (electron) moving with constant velocity $\mathbf{v}=v \mathbf{n}_{z}$ enters normally into a plate whose surfaces coincide with planes $z=-l$ and $z=0$. We assume that longitudinal ultrasonic vibrations are excited in the plate along the normal to its surface (along the axis $z$ ), that form a superlattice. The dielectric permittivity inside the plate we shall take in the form

$$
\varepsilon(z)=\varepsilon_{0}+\Delta \varepsilon \cos \left(k_{s} z+\omega_{s} t+\varphi\right),
$$

for $-l \leqslant z \leqslant 0$. In (11), $\omega_{s}, k_{s}$ are the cyclic frequency and the wave number of the ultrasound, $\varphi$ is the initial phase. Under the condition $\nu_{s} l / v \ll 1$, with $\nu_{s}=\omega_{s} /(2 \pi)$, during the transit time of the electron the dielectric constant in the superlattice is not notably changed. For relativistic electrons and for the plate thickness $l \lesssim 1 \mathrm{~cm}$ this leads to the constraint $\nu_{s} \ll 10^{11} \mathrm{~Hz}$. In the discussion below we shall assume that the plate is immersed in a homogeneous medium with dielectric permittivity $\varepsilon_{1}$.

We are interested in the radiation with frequencies $\omega$ satisfying the condition $\omega \gg k_{s} c$. The presence of small parameter $k_{s} c / \omega$ allows one to use the quasi-classical approximation for the evaluation of the radiation field. It is natural that in this case the plate boundaries $z=-l$ and $z=0$ must be sufficiently smooth. This condition is assumed to be observed [2] because the transition radiation on these boundaries is formed in a zone with macroscopic length. For the current density one has the expression

$$
\mathbf{j}=e v \delta(x) \delta(y) \delta(z-z(t)) \mathbf{n}_{z}, z(t)=-l+v\left(t-t_{0}\right) .
$$

In the Lorentz gauge, the vector potential of the electromagnetic field can be written as $\mathbf{A}=A \mathbf{n}_{z}$. This condition determines the radiation polarization. The magnetic field is perpendicular to the plane containing $\mathbf{n}_{z}$ and the photon wave vector.

In the quasi-classical approximation the Fourier component of the radiation field,

$$
A\left(k_{\perp}, \omega, z\right)=\int_{-\infty}^{+\infty} A(\mathbf{r}, t) e^{i\left(\omega t-k_{1} x-k_{2} y\right)} \frac{d x d y}{(2 \pi)^{3}}, \mathbf{k}_{\perp}=\left(k_{1}, k_{2}\right),
$$

in the region $z>0$ is determined by the expression [2]

$$
A\left(k_{\perp}, \omega, z\right)=\frac{i e V}{4 \pi^{2} c} \sqrt{\frac{\varepsilon(z)}{k_{3}(z)}} \int_{-\infty}^{+\infty} \exp \left[i \omega t+i \int_{z(t)}^{z} k_{3}\left(z^{\prime}\right) d z^{\prime}\right] \frac{d t}{\sqrt{k_{3}(z(t)) \varepsilon(z(t))}} .
$$

In formula (41) we have

$$
k_{3}(z)= \begin{cases}k_{3}^{(1)}, & z<-l, z>0 \\ k_{3}^{(0)}+a k_{s} \cos \left(k_{s} z+\varphi_{1}\right), & -l<z<0\end{cases}
$$

and $\varphi_{1}=\omega_{s} t_{0}+\varphi$. We have assumed that $\Delta \varepsilon$ is sufficiently small and the notations

$$
k_{3}^{(j)}=\sqrt{\omega^{2} \varepsilon_{j} / c^{2}-k_{\perp}^{2}}, j=0,1, a=\frac{\omega^{2} \Delta \varepsilon}{2 c^{2} k_{s} k_{3}^{(0)}},
$$


are introduced. Note that $a \sim \lambda_{s} \Delta \varepsilon / \lambda$, where $\lambda_{s}$ is the wavelength of the acoustic wave and $\lambda$ is the wavelength of the radiated photon.

By using (5) and the relation

$$
e^{i a \sin \tau}=\sum_{m=-\infty}^{+\infty} J_{m}(a) e^{i m \tau},
$$

where $J_{m}$ is the Bessel function of the first kind, after the integration for the vector potential in the region $z>0$ one finds

$$
\begin{aligned}
A\left(k_{\perp}, \omega, z\right)= & \frac{i e}{2 \pi^{2} c} \sqrt{\frac{\varepsilon_{1}}{k_{3}^{(1)}}} \exp \left[i \omega\left(t_{0}+l / v\right)+i k_{3}^{(1)} z\right] \\
& \times\left\{\frac{e^{i a \sin \left(\varphi_{1}\right)}}{\sqrt{k_{3}^{(0)} \varepsilon_{0}}} \sum_{m=-\infty}^{+\infty} J_{m}(a) e^{-i l\left(\omega / v-k_{3 m}^{(0)}\right) / 2-i m \varphi_{1}} \frac{\sin \left[l\left(\omega / v-k_{3 m}^{(0)}\right) / 2\right]}{\omega / v-k_{3 m}^{(0)}}\right. \\
& \left.+\frac{e^{i \phi_{1} / 2} \sin \left(\phi_{1} / 2\right)-e^{i \phi_{1}-i l\left(\omega / v-k_{3}^{(1)}\right) / 2} \sin \left[l\left(\omega / v-k_{3}^{(1)}\right) / 2\right]}{\sqrt{k_{3}^{(1)} \varepsilon_{1}}\left(\omega / v-k_{3}^{(1)}\right)}\right\} .
\end{aligned}
$$

Here we have used the notations

$$
\begin{aligned}
k_{3 m}^{(0)} & =k_{3}^{(0)}+m k_{s}, \\
\phi_{1} & =\left(k_{3}^{(0)}-k_{3}^{(1)}\right) l+a\left[\sin \left(\varphi_{1}\right)+\sin \left(k_{s} l-\varphi_{1}\right)\right] .
\end{aligned}
$$

The corresponding expressions for the electric and magnetic fields are found by using the standard formulae of electrodynamics.

\section{$3 \quad$ Radiation intensity}

Expression (8) may be used in calculations of the radiation intensity in the region $z>0$. We shall denote by $\theta$ the angle between the momentum of the radiated photon and the axis $z$. The energy radiated during the electron transit time in the range of frequencies $d \omega$ and angles $d \theta$ is determined by the relation [2]

$$
I(\omega, \theta) d \omega d \theta=(2 \pi)^{3} \frac{\varepsilon_{1}^{3 / 2} \omega^{4}}{c^{3}} \sin ^{3} \theta \cos ^{2} \theta\left|A\left(k_{\perp}, \omega, z \gg l\right)\right|^{2} d \omega d \theta,
$$

where $k_{\perp}=\left(\omega \sqrt{\varepsilon_{1}} / c\right) \sin \theta$. For the beam of relativistic electrons the expression $I(\omega, \theta)$ should be averaged over the phase $\varphi_{1}$ of particle flight into the plate. After this procedure, for the spectral-angular density of the radiated energy in the angular region $\sin \theta<\sqrt{\varepsilon_{0} / \varepsilon_{1}}$ we find

$$
\begin{aligned}
I(\omega, \theta)= & \frac{2 e^{2} \beta_{1}^{2}}{\pi c \sqrt{\varepsilon_{1}}} \sin ^{3} \theta \sum_{m=-\infty}^{+\infty} J_{m}^{2}\left(\frac{\omega \Delta \varepsilon}{2 c k_{s} \sqrt{\varepsilon_{0}-\varepsilon_{1} \sin ^{2} \theta}}\right) \\
& \times \sin ^{2}\left[\frac{l \omega}{2 v}\left(1-\beta_{1} \sqrt{\varepsilon_{0} / \varepsilon_{1}-\sin ^{2} \theta}+m k_{s} v / \omega\right)\right] \\
& \times\left[\frac{\sqrt{\varepsilon_{1} / \varepsilon_{0}}\left(\cos \theta / \sqrt{\varepsilon_{0} / \varepsilon_{1}-\sin ^{2} \theta}\right)^{1 / 2}}{1-\beta_{1} \sqrt{\varepsilon_{0} / \varepsilon_{1}-\sin ^{2} \theta}+m k_{s} v / \omega}-\frac{1}{1-\beta_{1} \cos \theta}\right]^{2},
\end{aligned}
$$


where $\beta_{1}=v \sqrt{\varepsilon_{1}} / c$ and $\varepsilon_{0}, \varepsilon_{1}$ are functions of $\omega$. Note that for relativistic electrons in this formula we have $k_{s} v / \omega \approx \lambda / \lambda_{s}$. For a given value of the radiation wavelength $\lambda=2 \pi c / \omega$, the peaks $\theta=\theta_{m}(\lambda)$ in the angular distribution of the radiation intensity are determined from the equation

$$
\sin ^{2} \theta_{m}(\lambda)=\frac{\varepsilon_{0}}{\varepsilon_{1}}-\frac{1}{\varepsilon_{1}}\left(\frac{c}{v}+m \frac{\lambda}{\lambda_{s}}\right)^{2} .
$$

The width of these peaks is of the order $\left(c / v+m \lambda / \lambda_{s}\right) \lambda /\left[l \sin \left(2 \theta_{m}\right)\right]$. For the radiation inside the plate the angular peaks in the radiation intensity are located at the outgoing photon angles $\theta=\theta_{m}^{(0)}(\lambda)=\arccos \left[\left(c / v+m \lambda / \lambda_{s}\right) / \sqrt{\varepsilon_{0}}\right]$. After the refraction on the boundary at $z=0$, the corresponding outgoing angle for the radiation in the region $z>0$ is determined by the relation $\sin \theta=\sqrt{\varepsilon_{0} / \varepsilon_{1}} \sin \left[\theta_{m}^{(0)}(\lambda)\right]$ which coincides with $\theta_{m}(\lambda)$ defined by (12).

The location of the peaks does not depend on the amplitude of the acoustic wave. The amplitude determines the heights of the peaks. The corresponding dependence is described by the Bessel functions in (11). Under the assumption $\lambda / \lambda_{s} \ll 1$, made above, at the peaks of the radiation corresponding to (12) the argument of the Bessel functions is approximately equal $\Delta \varepsilon \lambda_{s} /(2 \lambda)$. Hence, for given values of $\lambda, \lambda_{s}$, and $m$, the dependence of the peaks heights on the acoustic wave amplitude is described by the function $J_{m}^{2}\left(\Delta \varepsilon \lambda_{s} /(2 \lambda)\right)$. In particular, the maximum height of the peak is obtained for the amplitude of the acoustic wave corresponding to $\Delta \varepsilon \approx 2 x_{m} \lambda / \lambda_{s}$, where $x=x_{m}$ corresponds to the maximum of the function $J_{m}^{2}(x)$. For $m=0,1,2$ one has $x_{m}=0,1.84,3.05$, respectively. From the well-known properties of the Bessel functions it follows that the height of the central peak corresponding to $m=0$ decreases with increasing $\Delta \varepsilon$ in the range $0 \leqslant \Delta \varepsilon \leqslant 4.76 \lambda / \lambda_{s}$. The heights of the peaks generated by the acoustic wave increase with increasing $\Delta \varepsilon$ in the range $0 \leqslant \Delta \varepsilon \leqslant 3.68 \lambda / \lambda_{s}$ and $0 \leqslant \Delta \varepsilon \leqslant 6.1 \lambda / \lambda_{s}$ for $m=1$ and $m=2$ respectively. These features of the dependence of the radiation intensity on the acoustic wave amplitude will be illustrated below by a numerical example.

Let us consider special cases of formula (11). For X-ray transition radiation one has $\varepsilon_{0}=$ $1-\omega_{\mathrm{pl}}^{2} / \omega^{2}$ and formula (11) with $\varepsilon_{1}=1$ is reduced to the one derived in Ref. [15]. In the absence of the acoustic wave we have $\Delta \varepsilon=0$ and (11) takes the form

$$
\begin{aligned}
I_{0}(\omega, \theta)= & \frac{2 e^{2} \beta_{1}^{2}}{\pi c \sqrt{\varepsilon_{1}}} \sin ^{3} \theta \sin ^{2}\left[\frac{l \omega}{2 v}\left(1-\beta_{1} \sqrt{\varepsilon_{0} / \varepsilon_{1}-\sin ^{2} \theta}\right)\right] \\
& \times\left[\frac{\sqrt{\varepsilon_{1} / \varepsilon_{0}}\left(\cos \theta / \sqrt{\varepsilon_{0} / \varepsilon_{1}-\sin ^{2} \theta}\right)^{1 / 2}}{1-\beta_{1} \sqrt{\varepsilon_{0} / \varepsilon_{1}-\sin ^{2} \theta}}-\frac{1}{1-\beta_{1} \cos \theta}\right]^{2} .
\end{aligned}
$$

For a given $\omega$ the location of the peaks in the angular distribution of the radiation intensity is determined from the relation $\sin ^{2} \theta=\varepsilon_{0} / \varepsilon_{1}-\beta_{1}^{-2}$. It is of interest to note that the ongoing photon angle inside the plate corresponding to this $\theta$ is equal to the Cherenkov angle for the material of the plate: $\sin \theta=\sqrt{\varepsilon_{1} / \varepsilon_{0}} \sin \theta_{\mathrm{C}}$, where $\cos \theta_{\mathrm{C}}=c /\left(v \sqrt{\varepsilon_{0}}\right)$. As we see, for the appearance of the peaks in the forward direction we need to have the condition $c^{2} / v^{2} \leqslant \varepsilon_{0} \leqslant \varepsilon_{1}+c^{2} / v^{2}$. Otherwise, Cherenkov radiation emitted inside the plate will be reflected from the boundary. Formula (13) gives the quasi-classical approximation for the radiation intensity emitted by a charge flying into a dielectric plate in the direction of normal to its surface. The exact expression for the radiation intensity in this problem is given by Pafomov formula [17] (see also Refs. [2]-[7]). A detailed consideration of optical transition radiation described by Pafomov formula is recently given in Ref. [18. In this paper, in discussing the transition radiation in a dielectric plate, as a transparent material is taken glass. Numerical results based on Pafomov formula are given for the refractive index $n=1.5$. For this value of the refractive index Cherenkov radiation emitted in the plate is completely reflected from the boundary (see the condition given above). 
For a transparent material in the over-threshold case and under the condition $l \omega /(2 v) \gg 1$ the dominant contribution comes from the term in Pafomov formula with the resonant factor $\sin ^{2}[l \omega x /(2 v)] / x^{2}$, where $x=1-\beta_{1} \sqrt{\varepsilon_{0} / \varepsilon_{1}-\sin ^{2} \theta}$. Now, for simplicity considering the case $\varepsilon_{1}=1$, it can be seen that for a relativistic particle with $1-v / c \ll 1$ the radiation intensity near the Cherenkov peaks, corresponding to $\sin \theta=\sqrt{\varepsilon_{0}-c^{2} / v^{2}}$, is well approximated by formula (13) (we have checked this both analytically and numerically (see below) for fused quartz with the dispersion law given in the next section).

The Cherenkov peaks coincide with the $m=0$ peaks in the presence of the acoustic wave. As it follows from (11), the height of the peaks is reduced by the factor $J_{0}^{2}(a)$. For the parameters of the acoustic wave taken in accordance with $a=j_{0, s}, s=1,2, \ldots$, where $z=j_{0, s}$ are the zeros of the function $J_{0}(z)$, the corresponding radiation intensity vanishes. In the absence of acoustic waves the frequency range in which the peaks appear is determined by the condition $\varepsilon_{0} \leqslant \varepsilon_{1}+(c / v)^{2}$. When the acoustic waves are present the corresponding condition takes the form $\varepsilon_{0} \leqslant \varepsilon_{1}+\left(c / v+m \lambda / \lambda_{s}\right)^{2}$. The latter condition is less restrictive. In particular, we can have a situation when in the absence of the acoustic waves there are no peaks in a given frequency range and they appear in the presence of acoustic waves. Examples for this type of situation will be given below.

In the limit $l \rightarrow \infty$, by using the formula $\lim _{x \rightarrow \infty} \sin ^{2} \alpha x / x=\pi \alpha^{2} \delta(\alpha)$, for the spectralangular density of the radiation energy per unit length we find

$$
\begin{aligned}
I_{\infty}(\omega, \theta)= & \lim _{l \rightarrow \infty} I(\omega, \theta) / l=\frac{e^{2} \varepsilon_{1}^{3 / 2} \omega^{2}}{2 c^{3} \varepsilon_{0}} \sum_{m=-\infty}^{+\infty} J_{m}^{2}\left(\frac{\omega \Delta \varepsilon}{2 c k_{s} \sqrt{\varepsilon_{0}-\varepsilon_{1} \sin ^{2} \theta}}\right) \\
& \times \frac{\sin ^{3} \theta \cos \theta}{\sqrt{\varepsilon_{0} / \varepsilon_{1}-\sin ^{2} \theta}} \delta\left(\frac{\omega}{2 v}\left(1-\beta_{1} \sqrt{\varepsilon_{0} / \varepsilon_{1}-\sin ^{2} \theta}\right)+\frac{1}{2} m k_{s}\right) .
\end{aligned}
$$

As we see, in this case the radiation for a given $\theta$ has discrete spectrum determined by the relation

$$
\omega=\frac{-m k_{s} v}{1-\beta_{1} \sqrt{\varepsilon_{0} / \varepsilon_{1}-\sin ^{2} \theta}} .
$$

This relation directly follows from the periodicity properties of the problem. The spectral distribution of the radiation intensity is obtained from (14) by the integration over $\theta$ and in the case $\varepsilon_{1}=\varepsilon_{0}$ coincides with the result given in [2].

In the discussion above we have assumed that the particle moves with constant velocity along the direction perpendicular to the plate. In reality the particle trajectory will be affected by the interaction with atoms of medium (multiple scattering). It is known (see, for example, [2]) that the multiple scattering does not suppress the X-ray transition radiation. Moreover, it leads to the widening of the frequency range. An exact treatment for the influence of the multiple scattering on the intensity of radiation in the problem under consideration requires a separate consideration. We expect that the multiple scattering will result in the broadening of the radiation angular distribution for the peaks described above. By taking into account that the change in the medium density induced by acoustic wave is small, in the first approximation we can estimate this broadening by using the corresponding results for Cherenkov radiation in a homogeneous medium given in Ref. [19]. In this way, for the ratio of the angular broadening due to the multiple scattering to the angular width of the peaks estimated above we find $\approx l \sigma_{1} /\left(\pi \varepsilon_{0}\right)$, where $\sigma_{1}$ is defined by the mean squared angle of multiple scattering per unit length of the particle trajectory. Note that the characteristics of the peaks discussed above are determined by the relative velocity $v / c$ and for $v / c \approx 1$ they are relatively unsensitive to the energy of the 
particle, whereas the parameter $\sigma_{1}$ is decreasing with increasing energy. Hence, by increasing the particle energy we can reduce the relative role of the effects related to the multiple scattering.

\section{Numerical evaluation}

Here we are interested in the optical transition radiation. For the numerical evaluation of the radiation intensity the material of the plate should be specified. We assume that the plate is made of fused quartz. For the dielectric permittivity of fused quartz we use the Sellmeier dispersion formula

$$
\varepsilon_{0}=1+\sum_{i=1}^{3} \frac{a_{i} \lambda^{2}}{\lambda^{2}-l_{i}^{2}}
$$

where $a_{1}=0.6961663, a_{2}=0.4079426, a_{3}=0.8974794, l_{1}=0.0684043, l_{2}=0.1162414$, $l_{3}=9.896161$, and $\lambda$ is the wavelength of the radiation measured in micrometers. Formula (16) well describes the dispersion properties of fused quartz in the range $0.2 \mu \mathrm{m} \leqslant \lambda \leqslant 6.7 \mu \mathrm{m}$. Note that in this spectral region fused quartz is very weakly absorbing. For fused quartz the velocity of longitudinal ultrasonic vibrations is $\omega_{s} / k_{s} \approx 5.6 \times 10^{5} \mathrm{~cm} / \mathrm{s}$. In figures below we have plotted the spectral-angular density of the radiation intensity, $I(\omega, \theta) / \hbar$, for the energy of electrons equal to $2 \mathrm{MeV}$ and for the plate thickness $l=0.5 \mathrm{~cm}$. For the oscillation amplitude we have taken the value $\Delta n / n_{0}=0.05$, where $n_{0}$ is the number of electrons per unit volume for fused quartz.

As it is seen from the graphs given below the presence of the acoustic wave leads to the appearance of new peaks in both angular and spectral distributions of the radiation intensity. The height of the peaks can be tuned by choosing the parameters of the acoustic wave. In particular, the peak in the radiation intensity which is present in the absence of the acoustic wave is reduced by the factor $J_{0}^{2}(a)$, where $a$ is the argument of the Bessel function in (11). This peak can be completely removed by taking the parameters of the acoustic wave in such a way to have $a=j_{0, s}, s=1,2,3, \ldots$, where $z=j_{0, s}$ are the zeroes of the function $J_{0}(z)$. In a similar way other peaks can be suppressed by tuning the acoustic wave parameters. The graphs in figure 1 (full curves) are plotted for the frequency of acoustic wave $\nu_{s}=5 \mathrm{MHz}$. The dashed curves correspond to the radiation intensity in the absence of the acoustic wave. The left/right panel presents the angular/spectral distribution of the radiation intensity for a given frequency/angle. The parameters are chosen in a way to suppress the peak in the absence of the acoustic wave.

An example when the central peak is reduced partially is presented in figure 2. In order to illustrate the dependence of the radiation intensity on the amplitude of the ultrasonic vibrations, for this case, in figure 3 we plotted the angular distribution of the radiation intensity for $\Delta n / n_{0}=$ 0.025 (full curve) and $\Delta n / n_{0}=0.075$ (dashed curve). As it is seen, the height of the central peak decreases with increasing amplitude, whereas the heights for the peaks generated by the acoustic wave increase. This is in agreement with the features described in the previous section. For the values of the parameters corresponding to figures 2 and 3 we have also evaluated the radiation intensity near the Cherenkov peak in the absence of acoustic wave by using Pafomov formula. The corresponding results coincide with those evaluated within the framework of quasi-classical approximation with the relative accuracy more than $99 \%$.

In figure 4 we illustrate the suppression of the $m= \pm 1$ peaks in the case of acoustic wave with frequency $\nu_{s}=15 \mathrm{MHz}$. For the left (right) panel the height of the peak in the absence of the acoustic wave (dashed curves) is $\approx 9.5(\approx 11.4)$. In the absence of acoustic wave and for the energy of electron $\geqslant 2 \mathrm{MeV}$ the peaks in the forward direction appear in the range $\omega<9.62 \times 10^{14}$. In figure 5 we give an example when there is no peak in the absence of acoustic

wave and the peaks appear when the acoustic wave is excited. The presented graphs correspond 

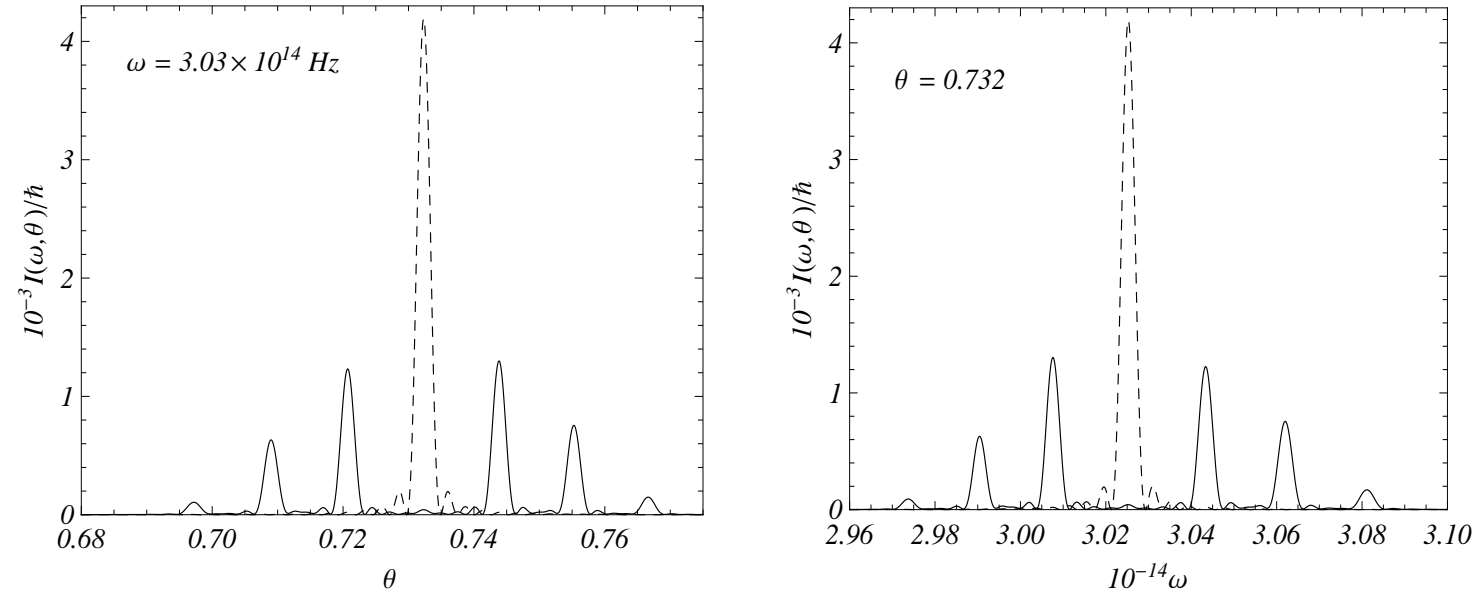

Figure 1: The angular (left panel) and spectral (right panel) distributions of the radiation intensity. The dashed curves correspond to the transition radiation when the acoustic wave is absent. For the left panel $\omega=3.03 \times 10^{14} \mathrm{~Hz}$ and for the right panel $\theta=0.732$. The frequency of the acoustic wave is equal to $5 \mathrm{MHz}$.
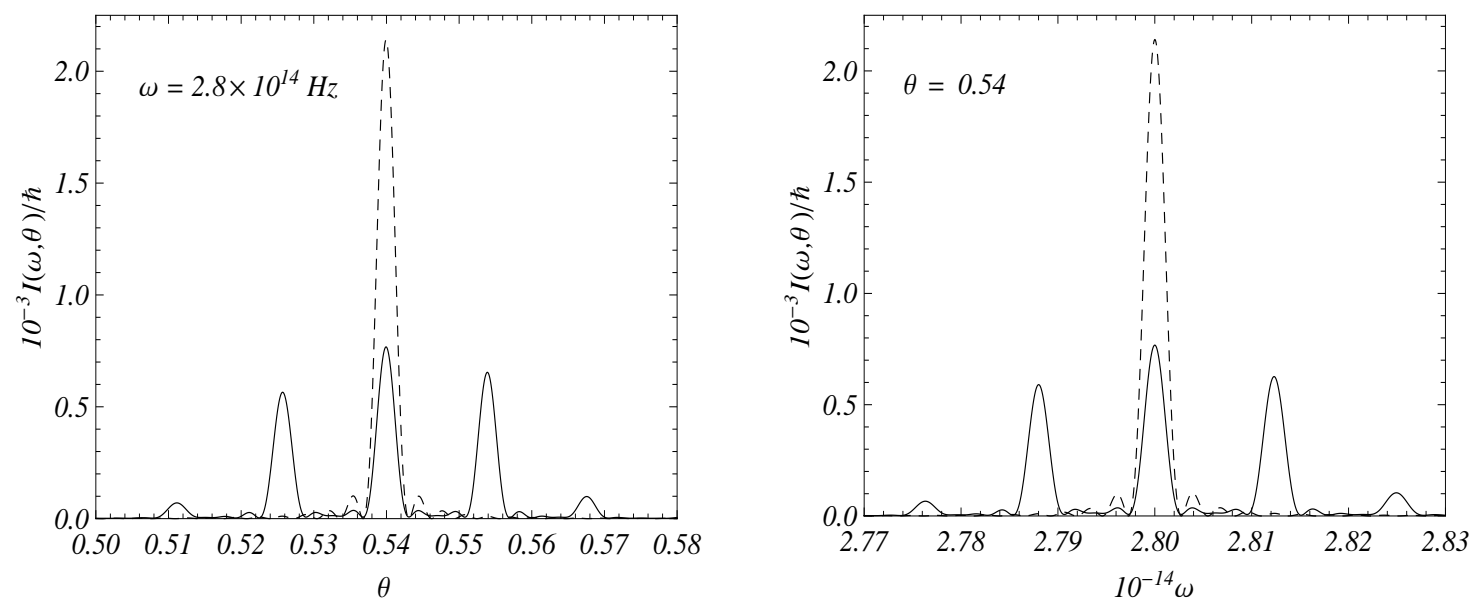

Figure 2: The same as in figure 1 for $\omega=2.8 \times 10^{14} \mathrm{~Hz}$ (left panel) and for $\theta=0.54$ (right panel). 


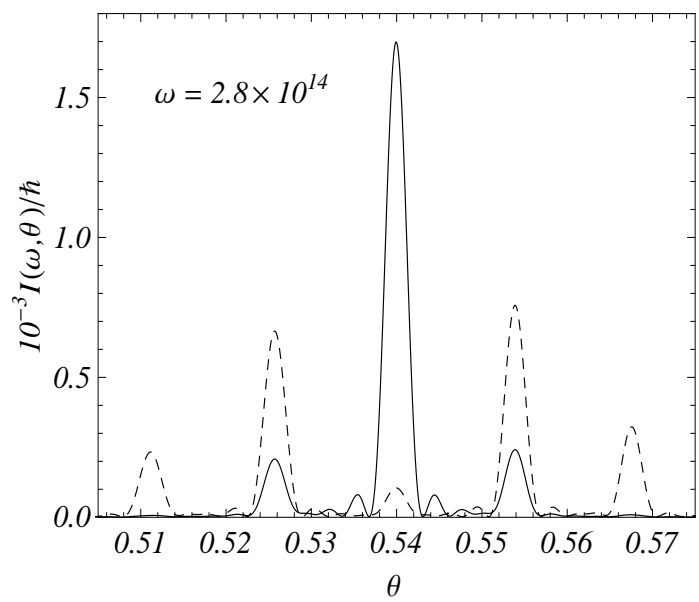

Figure 3: The same as in figure 2 for the amplitudes of acoustic wave corresponding to $\Delta n / n_{0}=$ 0.025 (full curve) and $\Delta n / n_{0}=0.075$ (dashed curve).

to the frequencies of acoustic wave $100 \mathrm{MHz}$ and $150 \mathrm{MHz}$ and to the frequency of the radiation $\omega=1 \times 10^{15} \mathrm{~Hz}$. Note that for all presented examples we have $\lambda \ll \lambda_{s}$ and the condition for the validity of the quasi-classical approximation is satisfied.
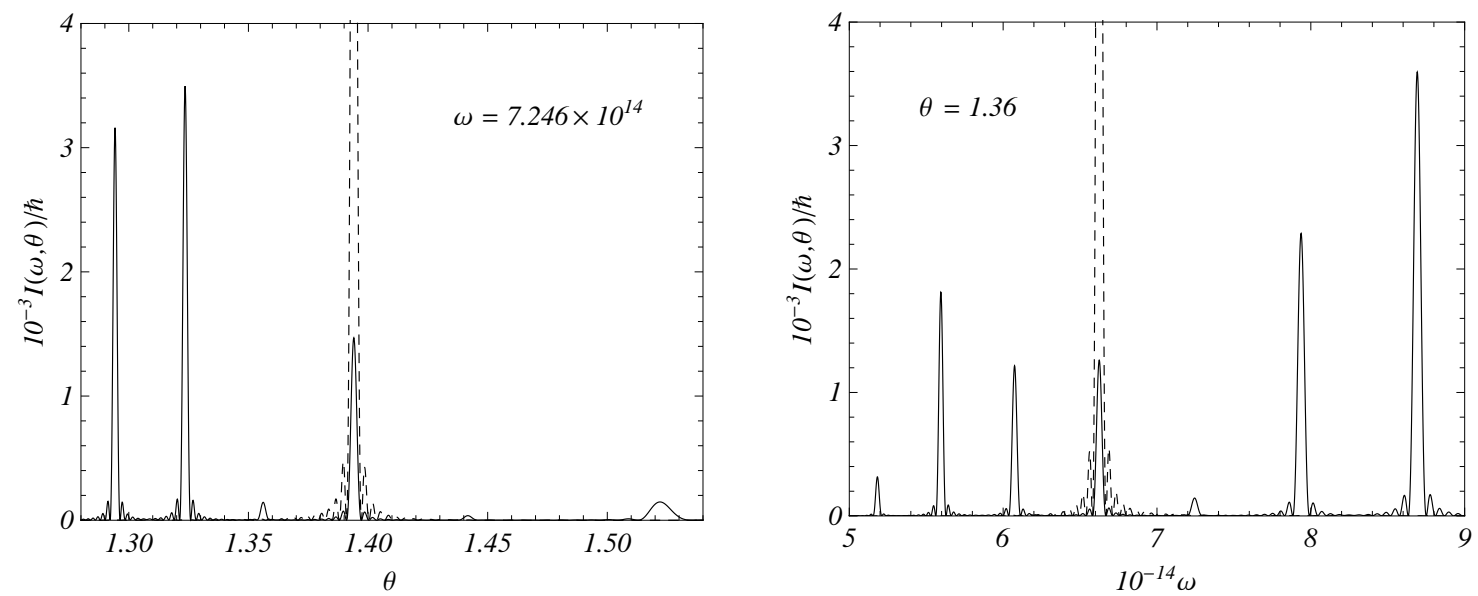

Figure 4: The same as in figure 1 for $\omega=7.246 \times 10^{14} \mathrm{~Hz}$ (left panel) and for $\theta=1.36$ (right panel). The frequency of the acoustic wave is equal to $15 \mathrm{MHz}$.

\section{Conclusion}

In the present paper we have investigated the transition radiation from an electron in a plate in the presence of acoustic waves. In the quasi-classical approximation we have derived formulae for the electromagnetic field and the radiation intensity in the forward direction. The spectralangular density of the radiated energy is given by formula (11). The numerical examples are given for a plate of fused quartz. These results show that the acoustic waves allow to control the parameters of the radiation. In particular, new resonance peaks appear in the spectral-angular distribution of the radiation intensity. The height of the peaks can be tuned by choosing the 


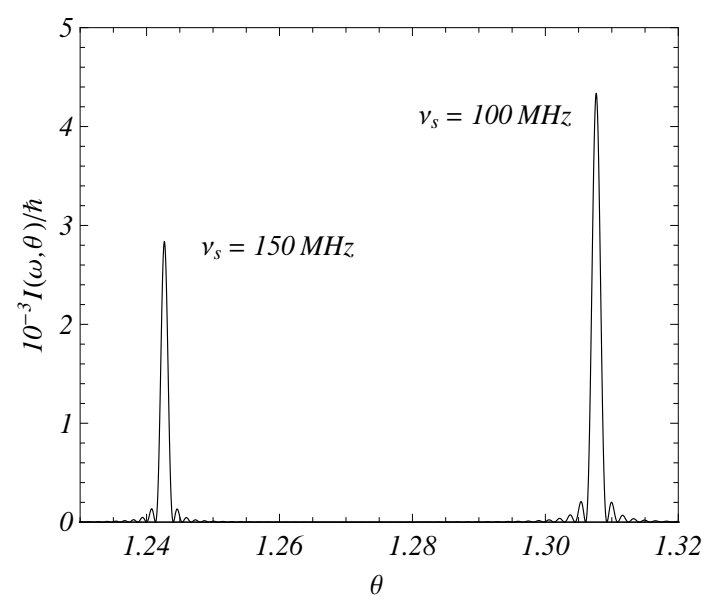

Figure 5: The angular distribution of the radiation intensity for $\omega=1 \times 10^{15} \mathrm{~Hz}$. The frequency of the acoustic wave is equal to $100 \mathrm{MHz}$ and $150 \mathrm{MHz}$.

parameters of the acoustic wave.

\section{References}

[1] A. H. Lumpkin, R. J. Dejus, N. S. Sereno, Phys. Rev. ST Accel. Beams 12, 040704 (2009).

[2] M. L. Ter-Mikaelian, High Energy Electromagnetic Processes in Condensed Media (Wiley Interscience, New York, 1972).

[3] G. M. Gharibian, S. Yan, Rentgenovskoye Perekhodnoye Izluchenie (Izd. AN Arm. SSR, Yerevan, 1983, in russian).

[4] V. L. Ginzburg, V. N. Tsytovich, Transition Radiation and Transition Scattering (Adam Hilger, Bristol, 1990).

[5] V. N. Baier, V. M. Katkov, V. M. Strakhovenko, Electromagnetic Processes at High Energies in Oriented Single Crystals (World Scientific, Singapore, 1998).

[6] P. Rullhusen, X. Artru, P. Dhez, Novel Radiation Sources Using Relativistic Electrons (World Scientific, Singapore, 1998).

[7] A. P. Potylitsin, Izluchenie Electronov v Periodicheskikh Structurakh (Izdatelstvo NauchnoTekhnicheskoi Literaturi, Tomsk, 2009, in russian).

[8] A. R. Mkrtchyan, L. Sh. Grigoryan, A. A. Saharian, A. N. Didenko, Acustica 75, 1984 (1991); A. A. Saharian, A. R. Mkrtchyan, L. A. Gevorgian, L. Sh. Grigoryan, B. V. Khachatryan, Nucl. Instrum. Methods B 173, 211 (2001).

[9] A. R. Mkrtchyan, H. A. Aslanyan, A. H. Mkrtchyan, R. A. Gasparyan, Phys. Lett. A 152, 297 (1991).

[10] A. R. Mkrtchyan, R. A. Gasparyan, R. G. Gabrielyan, Phys. Lett. A 115, 410 (1986); Phys. Lett. A 126, 528 (1988); L. Sh. Grigoryan et al., Nucl. Instrum. Methods B 173, 13 (2001); B 173, 184 (2001); L. Sh. Grigoryan, A. H. Mkrtchyan, H. F. Khachatryan, 
V. U. Tonoyan, W. Wagner, Nucl. Instrum. Methods B 201, 25 (2003); W. Wagner, B. Azadegan, L. Sh. Grigoryan, J. Pawelke, Europhys. Lett. 78, 56004 (2007); B. Azadegan, L. Sh. Grigoryan, J. Pawelke, W. Wagner, J. Phys. B 41, 235101 (2008).

[11] A. R. Mkrtchyan, A. A. Saharian, L. Sh. Grigoryan, B. V. Khachatryan, Mod. Phys. Lett. A 17, 2571 (2002); A. R. Mkrtchyan, A. A. Saharian, V. V. Parazian, Mod. Phys. Lett. B 20, 1617 (2006); V. V. Parazian, J. Phys.: Condens. Matter 21, 185401 (2009).

[12] A. A. Saharian, A. R. Mkrtchyan, V. V. Parazian, L. Sh. Grigoryan, Mod. Phys. Lett. A 19, 99 (2004); N. F. Shulga, V. V. Boyko, JETP Lett. 84, 365 (2006); A. R. Mkrtchyan, A. A. Saharian, V. V. Parazian, Mod. Phys. Lett. B 23, 2573 (2009).

[13] A. V. Korol, A. V. Solov'yov, W. Greiner, J. Phys. G: Nucl. Part. Phys. 24, L45 (1998); A. V. Korol, A. V. Solov'yov, W. Greiner, Int. J. Mod. Phys. E 8, 49 (1999); R. O. Avakian, L. A. Gevorgian, K. A. Ispirian, R. K. Ispirian, JETP Lett. 68, 467 (1998); Q.-R. Zhang, Int. J. Mod. Phys. E 8, 493 (1999); U. Mikkelsen, E. Uggerhøj, Nucl. Instrum. Methods B 160, 435 (2000); A. V. Korol, A. V. Solov'yov, W. Greiner, J. Phys. G: Nucl. Part. Phys. 27, 95 (2001); R. O. Avakian, L. A. Gevorgian, K. A. Ispirian, R. K. Ispirian, Nucl. Instrum. Methods B 173, 112 (2001); A. V. Korol, W. Krause, A. V. Solov'yov, W. Greiner, Nucl. Instrum. Methods A 483, 455 (2002); R. O. Avakian, K. T. Avetyan, K. A. Ispirian, E. G. Melikyan, Nucl. Instrum. Methods A 508, 496 (2003); S. Bellucci et al, Phys. Rev. Lett. 90, 034801 (2003); S. Bellucci, Mod. Phys. Lett. B 19, 85 (2005); S. Bellucci, V. A. Maisheev, Nucl. Instrum. Methods B 252, 339 (2006).

[14] A. V. Korol, A. V. Solov'yov, W. Greiner, Int. J. Mod. Phys. E 13, 867 (2004).

[15] L. Sh. Grigoryan, A. H. Mkrtchyan, A. A. Saharian, Nucl. Instrum. Methods B 145, 197 (1998).

[16] L. Sh. Grigoryan, A. R. Mkrtchyan, H. F. Khachatryan, S. R. Arzumanyan, W. Wagner, arXiv:0911.3494.

[17] V. E. Pafomov, Proc. FIAN USSR 44, 90 (1969).

[18] A. Hrmo, J Ruzicka, Nucl. Instrum. Methods A 451, 506 (2000).

[19] V. M. Grichine, Nucl. Instrum. Methods A 563, 364 (2006). 